Accepted for Publication in the Astronomical Journal

Preprint typeset using $\mathrm{AT}_{\mathrm{E}} \mathrm{X}$ style emulateapj v. 5/2/11

\title{
TIME-SERIES SPECTROSCOPY OF TWO CANDIDATE DOUBLE DEGENERATES IN THE OPEN CLUSTER
} NGC 6633

\author{
Kurtis A. Williams \\ Department of Physics \& Astrophysics, Texas A\&M University-Commerce, P.O. Box 3011, Commerce, TX, 75429, USA \\ Donald Serna-Grey ${ }^{1}$ \\ Department of Astronomy, University of Washington, Box 351580, Seattle, WA, 98195, USA \\ Subho Chakraborty ${ }^{2}$ \\ Department of Physics \& Astrophysics, Texas A\&M University-Commerce, P.O. Box 3011, Commerce, TX, 75429, USA
}

\section{A. Gianninas and Paul A. Canton}

Homer L. Dodge Department of Physics and Astronomy, University of Oklahoma, 440 W. Brooks St., Norman, OK, 73019, USA Accepted for Publication in the Astronomical Journal

\begin{abstract}
Type Ia supernovae are heavily used tools in precision cosmology, yet we still are not certain what the progenitor systems are. General plausibility arguments suggest there is potential for identifying double degenerate Type Ia supernova progenitors in intermediate-age open star clusters. We present time-resolved high-resolution spectroscopy of two white dwarfs in the field of the open cluster NGC 6633 that had previously been identified as candidate double degenerates in the cluster. However, three hours of continuous observations of each candidate failed to detect any significant radial velocity variations at the $\gtrsim 10 \mathrm{~km} \mathrm{~s}^{-1}$ level, making it highly unlikely that either white dwarf is a double degenerate that will merge within a Hubble Time. The white dwarf LAWDS NGC 66334 has a radial velocity inconsistent with cluster membership at the $2.5 \sigma$ level, while the radial velocity of LAWDS NGC 66337 is consistent with cluster membership. We conservatively conclude that LAWDS 7 is a viable massive double degenerate candidate, though unlikely to be a Type Ia progenitor. Astrometric data from GAIA will likely be needed to determine if either white dwarf is truly a cluster member.

Subject headings: binaries: close — binaries: spectroscopic — open clusters and associations: individual (NGC 6633) — supernovae: general — white dwarfs
\end{abstract}

\section{INTRODUCTION}

The double-degenerate (DD) channel for Type Ia supernovae proposes that two carbon-oxygen core white dwarfs (WDs) with a total mass at or above the Chandrasekhar mass, $M_{\mathrm{Ch}}$, will spiral inwards due to gravitational radiation, merge, and explode (Iben \& Tutukov 1984). The degree to which the DD channel contributes to Type Ia supernovae is still a matter of vociferous debate (e.g., Maoz et al. 2014; Santander-García et al. 2015; Garcia-Berro et al. 2015). We present observations of two candidate DDs in an intermediate-age open star cluster that, while not constraining the DD channel any further, illustrate the potential utility of WDs in open star clusters as probes of the DD channel.

Observational and theoretical arguments from several groups suggest that some fraction of Type Ia supernovae come from a "prompt" population, with supernova onset $\lesssim 500$ Myr after a star formation event, as well as a second progenitor population with a much longer delay before detonation (e.g., Barris \& Tonrv 2006; Mannucci et al. 2005; Scannapieco \& Bildsten 2005;

\footnotetext{
Kurtis.Williams@tamuc.edu

${ }^{1}$ REU Participant, Department of Physics \& Astrophysics, Texas A\&M University-Commerce, Commerce, TX

${ }^{2}$ Current address: The Graduate Center, City University of New York, New York, NY, USA
}

Sullivan et al. 2006; Maoz et al. 2010, 2011). Therefore, stellar populations with ages $\lesssim 500 \mathrm{Myr}$ should contain more prompt Type Ia progenitors, whatever these progenitor systems may actually be.

Regardless of the delay time, the DD Type Ia progenitor channel requires at least one of the WDs to have a mass $\gtrsim M_{\mathrm{Ch}} / 2 \approx 0.7 M_{\odot}$. Based on the semiempirical initial-final mass relation, such a WD will result from a progenitor star with $M_{\text {init }} \gtrsim 2.8 M_{\odot}$ (e.g., Catalán et al. 2008; Williams et al. 2009; Dobbie et al. 2009, 2012; Zhao et al.|2012; Cummings et al.|2015) and with a nuclear lifetime of $\lesssim 560 \mathrm{Myr}$, assuming metallicity $Z=0.019$ (Marigo et al. 2008), consistent with the delay time discussed in the previous paragraph. Given the known scatter in the initial-final mass relation around $\sim 3 M_{\odot}$, we emphasize that these numbers are intended to be estimates only.

The upper limits on orbital periods producing a DD merger within a Hubble time require that Type Ia progenitors must go through a common envelope phase. It has been observed that the binary frequency of stars rises from late-type B stars through $\mathrm{O}$ stars $\left(M_{*} \gtrsim 3 M_{\odot}\right)$, and that these massive binaries are far more likely to have mass ratios closer to unity than expected from random chance (e.g., Lucv \& Ricco 1979; Pinsonneault \& Stanek 2006; Duchêne \& Kraus 2013). Since at least one of the 
members of a DD must have originated from a star of spectral type late B or earlier in order to produce a WD of mass $\geq 0.7 M_{\odot}$, it is plausible that both degenerates in any $\mathrm{D} \overline{\mathrm{D}}$ Type Ia progenitor system evolved from stars with main sequence masses $\gtrsim 3 M_{\odot}$, which have nuclear lifetimes of $\lesssim 500$ Myr.

Taken together, these models and observations suggest that DD Type Ia supernova progenitors, if they exist, could form within $\sim 500 \mathrm{Myr}$ of the onset of star formation. Therefore, a simple stellar population of age $\lesssim 500$ Myr, such as open star clusters with main sequence turnoff masses $\gtrsim 3 M_{\odot}$, may be relatively fertile ground for hunting DD Type Ia progenitor systems. If the DD systems have not already merged and detonated as prompt Type Ia supernovae prior to our observations, the individual WD components should be relatively bright and hot, and thus straightforward to identify in intermediate-age open star clusters with existing ground-based facilities.

\subsection{Two Candidate Double Degenerates in the Field of NGC 6633}

For the past several years, we have engaged in studies of WDs in intermediate-age open clusters, with goals of observationally constraining the relationship between WD masses and their progenitor star masses (the initialfinal mass relation) as well as the maximum mass of WD progenitors (Williams 2002; Williams et al. 2004; Liebert et al. 2005; Williams \& Bolte 2007; Rubin et al. 2008; Williams et al. 2009; Dobbie et al. 2009, 2012; Liebert et al. 2013).

One necessary step in this process is identifying confirmed WDs as cluster members. The vast majority of the WDs we have discovered do not have existing proper motion measurements precise enough for cluster membership determinations. Instead, we have constrained cluster membership via a version of spectroscopic parallax described in detail in Williams \& Bolte (2007) and Williams et al. (2009). In summary, we obtain absolute magnitudes for each WD via $T_{\text {eff }}$ and $\log g$ spectral fits of observed spectra to model atmospheres combined with WD evolutionary models that include the mass-radius relation. In this paper, we use the color and model calculations from Holberg \& Bergeron (2006); Kowalski \& Saumon (2006); Tremblay et al. (2011); and Bergeron et al. (2011) 3 .

We then subtract the apparent magnitude obtained via optical photometry from the derived absolute magnitude to obtain the apparent distance modulus of the WD. If the apparent distance modulii of the WD and star cluster are consistent, and if the WD cooling age is less than the cluster age, then we identify the WD as a likely cluster member. This greatly reduces field contamination in the WD sample, but it precludes the inclusion of WDWD binary systems if the secondary star contributes any significant flux.

In Williams \& Bolte (2007), we confirmed the presence of 13 WDs in the field of the open cluster NGC 6633 . NGC 6633 has an age of $\approx 560$ Myr (Williams \& Bolte 2007), a slightly subsolar metallicity $[\mathrm{Fe} / \mathrm{H}] \approx-0.1$, a distance modulus $(m-M)_{0}=8.01 \pm 0.09$, and moderate

3 Available online at http://www . astro.umontreal.ca/ bergeron/CoolingModels/ reddening $E(B-V)=0.165 \pm 0.011$ (Jeffries et al. 2002).

Based on the Williams \& Bolte (2007) analysis, only one WD (LAWDS NGC 6633 27) is consistent with the apparent distance modulus of $(m-M)_{V}=8.52 \pm$ 0.10 (assuming $R_{V}=3.1$ ). However, two additional WDs (LAWDS NGC 66334 and LAWDS NGC 6633 7, hereafter LAWDS 4 and LAWDS 7, respectively) are significantly more massive $\left(\geq 0.8 M_{\odot}\right)$ than the mode of the field WD mass distribution $\left(\approx 0.65 M_{\odot}\right.$ Tremblay \& Bergeron 2009). Additionally, both have fluxes almost exactly a factor of two higher than would be expected for cluster members, i.e., apparent distance moduli $0.75 \mathrm{mag}$ less than the cluster. Astrometric and photometric data for these two objects are given in Table 11.

Proper motion cluster memberships of these two stars using astrometric data from the USNO-B catalog (Monet et al. 2003) are inconclusive. The proper motion vector $\left(\mu_{\mathrm{RA}}, \mu_{\text {Decl }}\right)$ of NGC 6633 is $(0.1 \pm 0.22,-2.00 \pm$

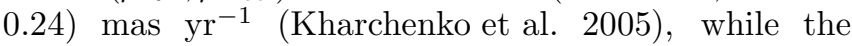
surounding fields stars have a mean proper motion vector of $(-2.06,-5.58)$ mas $\mathrm{yr}^{-1}$ with a dispersion of $(20.5,20.8)$ mas $\mathrm{yr}^{-1}$ (Dias et al. 2014). LAWDS 4 has a right ascension proper motion formally inconsistent with the cluster at the $4 \sigma$ level, but USNO-B catalog proper motions of bright QSOs show an rms dispersion of

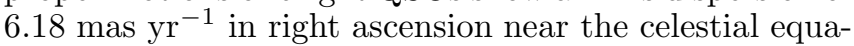
tor (Munn et al. 2004), essentially negating this result. LAWDS 7 has no detected proper motion in the USNO-B catalog. These results are not surprising as proper motion is found to bee a poor discriminator for faint stars in this field; the mean cluster tangential velocity is only $\sim 1.5 \mathrm{~km} \mathrm{~s}^{-1}$ compared to the field (Jeffries 1997).

Simulations of star cluster evolution suggest binary WD cooling sequences should exist at roughly this overluminosity (e.g., Hurley \& Shara 2003; Geller et al. 2013), and features in the WD luminosity function of the rich open cluster NGC 6791 can be interpreted as a binary cooling sequence (e.g., García-Berro et al. 2011), though a He-core WD cooling sequence also explains these observations (Kalirai et al. 2007). We therefore suggested in Williams \& Bolte (2007) that LAWDS 4 and LAWDS 7 may be double degenerates.

Since our initial publication, other open cluster WD studies have flagged WDs with distance moduli $\leq 0.75$ mag foreground to their cluster as candidate DDs (e.g. Kalirai et al. 2008; Rubin et al. 2008), though none of the proposed candidates are so enticingly close to the $0.75 \mathrm{mag}$ over-luminosity often indicative of binary systems. If LAWDS 4 and LAWDS 7 are equal-mass DDs, each system's total mass is super-Chandrasekhar. Based on these arguments, we proposed for and received time at the Keck Observatory through the NOAO TSIP program (NOAO proposal 08A-0124) to perform follow-up spectroscopy on these two candidate double degenerate systems.

\section{REVISED WHITE DWARF PARAMETERS}

Our WD parameters in Williams \& Bolte (2007) are based on Balmer line fits using slightly modified versions of synthetic, pure-H atmospheres used in Finley et al. (1997) and graciously provided by D. Koester at the time. Since that time, the inclusion of additional physics and of new calculations of quantum effects and Stark broad- 
TABLE 1

Astrometric and Photometric Parameters of the Candidate Double Degenerates

\begin{tabular}{ccccccc}
\hline \hline Object & $\begin{array}{c}\text { R.A. } \\
(\mathrm{J} 2000)\end{array}$ & $\begin{array}{c}\text { Decl. } \\
(\mathrm{J} 2000)\end{array}$ & $\begin{array}{c}\mu_{\mathrm{RA}} \\
(\mathrm{mas} / \mathrm{yr})\end{array}$ & $\begin{array}{c}\mu_{\text {Decl }} \\
(\mathrm{mas} / \mathrm{yr})\end{array}$ & $\begin{array}{c}V \\
(\mathrm{mag})\end{array}$ & $\begin{array}{c}B-V \\
(\mathrm{mag})\end{array}$ \\
\hline LAWDS 4 & 182710.4 & 062615.7 & $-8.0 \pm 2.0$ & $6.0 \pm 5.0$ & $18.82 \pm 0.02$ & $0.16 \pm 0.03$ \\
LAWDS 7 & 182749.9 & 062051.8 & $\cdots$ & $\cdots$ & $19.27 \pm 0.02$ & $0.23 \pm 0.03$
\end{tabular}

Note. - Proper motions are from Monet et al. (2003); other data are from Williams \& Bolte (2007).

ening has led to small but systematic shifts in $T_{\text {eff }}$ and $\log g$ determinations for DA WDs (Tremblay \& Bergeron 2009; Koester et al. 2009; Koester 2010). Under the assumption that the resulting new atmospheric models and parameters are more accurate than the earlier models, we have recalculated the surface gravity and effective temperatures for LAWDS 4 and LAWDS 7.

We simultaneously fit the Balmer lines to model spectra using the spectroscopic technique developed by Bergeron et al. (1992); we applied the recent innovations and techniques presented in Gianninas et al. (2011) and references therein. We first normalized each individual Balmer line such that the continuum level was set to unity in both the observed and model spectra. We compared the normalized observed spectra with the normalized synthetic spectra after convolving them with a Gaussian function approximating the instrumental profile. Using the steepest descent nonlinear least-squares method of Levenberg-Marquardt (Press et al. 1992), we determined $T_{\text {eff }}$ and $\log g$ from the grid of model spectra. We did not apply the 3D convective model corrections of Tremblay et al. (2013), as LAWDS 4 and LAWDS 7 are sufficiently hot that their atmospheres are radiative, and the corrections would be negligible.

The new WD parameters, along with the derived WD masses, cooling times, and distance moduli are given in Table 2. A color-magnitude diagram for the cluster, with the two DD candidates indicated by open squares, can be found in Figure 6 of Williams \& Bolte (2007). Figures 8a and $8 \mathrm{~b}$ of Williams \& Bolte (2007) show how the physical parameters of these two WDs compare to those expected for cluster members with the same apparent magnitudes; the new physical parameters shift these stars to slightly higher temperatures. The newer WD masses and distance moduli are consistent with the earlier published values, and these two WDs still stand out as candidate DDs.

\section{HIGH-RESOLUTION SPECTROSCOPY}

WDs of spectral type DA often exhibit sharp, narrow line cores superimposed on the broad Balmer absorption lines. These cores are attributed to non-local thermodynamical equilibrium (NLTE) conditions in the WD atmosphere (Greenstein \& Peterson 1973). The goal of our new observations was to obtain time-series spectroscopy with sufficient resolution to detect the NLTE core of $\mathrm{H} \alpha$, similar to methods employed by the European Southern Observatory SN Ia Progenitor Survey (Napiwotzki et al. 2001). Since we had only one short summer night for observations, we observed each target for only $3 \mathrm{~h}$. Although DD Type Ia progenitors can have orbital periods of up to 12 hours, detection of even a partial orbit should be sufficient to warrant additional observing time.

We obtained data with the Echellette Spectrograph and Imager (ESI, Sheinis et al. 2002) on Keck II on UT $2008 \mathrm{Jul} 3$. We operated the instrument in echelle mode with a slit width of $0 . \prime 75$. We measured the velocity resolution using the full-width at half-maximum (FWHM) of the $5577 \AA$ night sky line at $55.7 \mathrm{~km} / \mathrm{s}$, consistent with the stated instrumental resolution of $55.9 \mathrm{~km} / \mathrm{s}(\approx 5$ pix $)$. The weather was photometric with steady seeing of 0 !' 6 . For exposures of LAWDS 7, one mirror segment did not align properly, leading to a faint second image in the spatial direction; we ignored this second image in our further analysis.

We varied exposure times for LAWDS 4 in hopes of detecting any possible velocity smearing resulting from orbital periods $\lesssim 20$ minutes. This resulted in signalto-noise $(\mathrm{S} / \mathrm{N})$ ratios per pixel ranging from $13(600 \mathrm{~s}$ exposures) to 21 (1200 s exposures) in the continuum surrounding the the $\mathrm{H} \alpha$ line. Exposure times for LAWDS 7 were held constant after the first exposure due to the lower flux from this fainter star, with $\mathrm{S} / \mathrm{N} \approx 18 \mathrm{pix}^{-1}$. Details on each target exposure are presented in Table 3 for LAWDS 4 and Table 4 for LAWDS 7.

We reduced the data using the ESIRedux package of Prochaska et al. (2003) and followed the basic recipe for reductions given in the ESIRedux Cookbool 1 . ESIRedux applies corrections to the data from bias images and flat fields, extracts the object spectrum, subtracts background sky, wavelength calibrates the extracted spectra, and uses night sky lines to correct the wavelength zero points for any instrumental flexure remaining in each individual exposure.

We applied bias frames and $\mathrm{HgNe}$ and $\mathrm{CuAr}$ arc lamp solutions obtained during the run. ESIRedux was not able to use our internal flat fields, so we used an archived dome flat provided with the ESIRedux code for flat fielding; no obvious flat fielding errors remained in the spectra. We also used an archived map of the order curvature provided with the ESIRedux code for the spectral tracing and extraction; again, the results are satisfactory.

We used exposures of Feige 67 to apply a relative flux correction. The orders were stitched together to produce a one-dimensional output spectrum; wavelengths are calibrated to vacuum wavelengths. The resulting spectra are of high quality for $4000 \AA \leq \lambda \leq 8000 \AA$, though residual noise from the regions of order overlap were visible. We confirmed the wavelength solutions by measuring centroids of strong night-sky emission lines; these centroids are consistent with zero velocity with a

4 Available at http://www2.keck.hawaii.edu/inst/esi/ESIRedux/esi_cookbook.html 
TABLE 2

Revised Atmospheric Parameters for LAWDS 4 and LAWDS 7

\begin{tabular}{cccccccc}
\hline \hline Object & $\begin{array}{c}T_{\text {eff }} \\
(\mathrm{K})\end{array}$ & $\log g$ & $\begin{array}{c}M_{\mathrm{WD}} \\
\left(M_{\odot}\right)\end{array}$ & $\begin{array}{c}\tau_{\text {cool }} \\
(\mathrm{Myr})\end{array}$ & $\begin{array}{c}M_{V} \\
(\mathrm{mag})\end{array}$ & $\begin{array}{c}(m-M)_{V} \\
(\mathrm{mag})\end{array}$ & $\begin{array}{c}\Delta\left[(m-M)_{V}\right]^{\mathrm{a}} \\
(\mathrm{mag})\end{array}$ \\
\hline LAWDS 4 & $21800 \pm 350$ & $8.29 \pm 0.05$ & $0.80 \pm 0.03$ & 98.8 & $11.07 \pm 0.09$ & $7.74 \pm 0.09$ & $-0.78 \pm 0.13$ \\
LAWDS 7 & $19360 \pm 310$ & $8.42 \pm 0.05$ & $0.88 \pm 0.03$ & 187 & $11.48 \pm 0.09$ & $7.78 \pm 0.09$ & $-0.74 \pm 0.13$
\end{tabular}

a Difference of the WD and NGC 6633 distance modulii

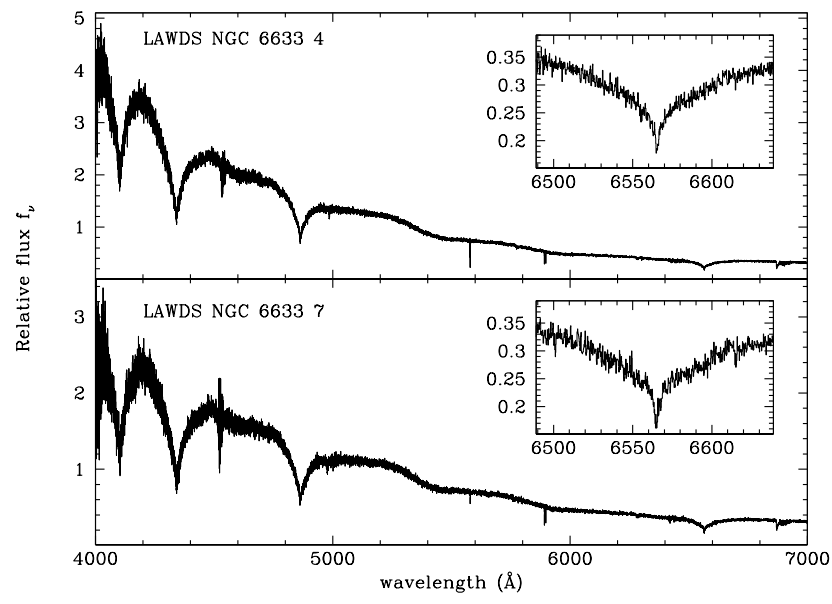

FIG. 1.- Coadded ESI spectra for LAWDS 4 (top) and LAWDS 7 (bottom). Insets show the central regions of the $\mathrm{H} \alpha$ absorption lines and the NLTE cores. Narrow interstellar Na D lines and telluric B-band absorption are visible, as are residuals from $5577 \AA$ night sky line subtraction. Most other high-frequency features (such as the wiggles at $4500 \AA$ and $6400 \AA$ ) are due to the order overlap regions. Both spectra qualitatively look like typical single DA white dwarfs.

dispersion of less than $1 \mathrm{~km} \mathrm{~s}^{-1}$. Due to this, we did not apply corrections from a radial velocity standard star. Further, as we were interested primarily in the Balmer absorption lines, we did not apply telluric corrections to the data.

Figure 1 shows the co-added spectra from all exposures of each target. In addition to the broad Balmer absorption lines, narrow $\mathrm{Na} \mathrm{D}$ lines are observed; these are presumably interstellar in origin.

Figure 2 shows the central regions of the $\mathrm{H} \alpha$ and $\mathrm{H} \beta$ lines for LAWDS 4 and LAWDS 7, as well as of the known short period DD HE 2209-1444, the data for which were obtained on the same night with the same instrumental configuration, but at a random phase angle. This illustrates what a double-lined spectroscopic binary would look like in our data, given sufficient signal-to-noise. Visual inspection showed evidence for only one NLTE core in the $\mathrm{H} \alpha$ line of LAWDS 4.

The $\mathrm{H} \alpha$ and $\mathrm{H} \beta$ cores of LAWDS 7 exhibit one obvious NLTE core and evidence of a second absorption feature redward of the strong core. This second feature is qualitatively similar to the weaker NLTE core in the spectrum of HE 2209-1444. However, the centroids of the redder features differ in the two lines - in $\mathrm{H} \alpha$, the second dip is at a velocity of $\approx+130 \mathrm{~km} \mathrm{~s}^{-1}$, while in $\mathrm{H} \beta$ it appears at a velocity of $\approx 200 \mathrm{~km} \mathrm{~s}^{-1}$. Further, features with similar depths appear elsewhere in the lines (e.g., at +250 $\mathrm{km} \mathrm{s}^{-1}$ in LAWDS $7 \mathrm{H} \beta$, and at $-80 \mathrm{~km} \mathrm{~s}^{-1}$ in LAWDS $4 \mathrm{H} \alpha$ ). Therefore we consider these features intruiging,

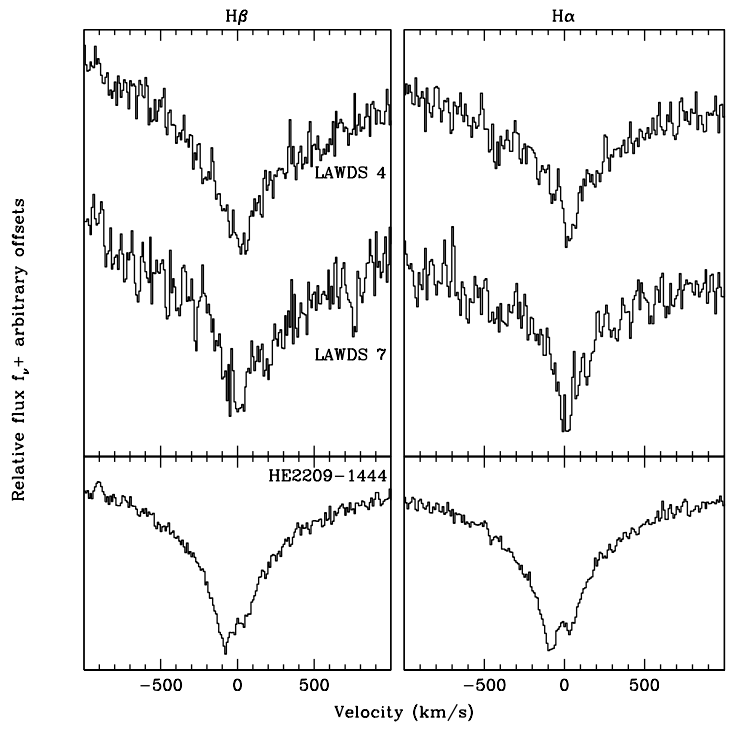

Fig. 2.- The $\mathrm{H} \beta$ (left) and $\mathrm{H} \alpha$ (right) absorption line centers for LAWDs 4 and 7 (top panel) and the known double degenerate HE 2209-1444, obtained with the same instrumental configuration at a random phase angle. The two NLTE cores are visible in HE $2209-1444$, but only a single line core is visible in LAWDS 4. LAWDS 7 has a feature suspiciously similar to the second cores in HE 2209-1444, but we cannot consider this significant; see the discussion in the text.

but they are neither significant nor convincingly a second NLTE core. Further, given the 3-hour total integration time of the coadded spectra, one would expect radial velocity smearing in the line cores, but such smearing is not consistent with the measured FWHMs.

We note that the NLTE cores can vary in strength or even appear in emission depending on the atmospheric parameters and structures. The lack of a 2nd NLTE core does not, therefore, preclude the system from being a double-lined spectroscopic binary if such emission has filled in the line core.

\subsection{Time-series radial velocities}

We search for radial velocity variations using the individual spectral exposures listed in Tables 3 and 4. Unfortunately, the NLTE $\mathrm{H} \alpha$ core is weak in individual exposures. We should have expected this given the moderate $T_{\text {eff }}$ of our candidates; the $\mathrm{H} \alpha$ NLTE core becomes less well defined at higher $T_{\text {eff }}$ in DA WDs.

After we unsuccessfully attempted several methods of measuring robust radial velocities from the $\mathrm{H} \alpha$ and $\mathrm{H} \beta$ NLTE line cores alone (Chakraborty 2013), we changed our approach to utilize larger portions of the spec- 
TABLE 3

OBSERVATions AND RADial Velocity Data For LAWDS 4

\begin{tabular}{lccc}
\hline \hline $\begin{array}{l}\text { Exposure } \\
\text { Number }\end{array}$ & $\begin{array}{c}T_{\text {mid }} \\
(\text { HJD-2454650.5) }\end{array}$ & $\begin{array}{c}t_{\exp } \\
(\mathrm{s})\end{array}$ & $\begin{array}{c}v_{\text {rel }} \\
\left(\mathrm{km} \mathrm{s}^{-1}\right)\end{array}$ \\
\hline 1 & 0.29067 & 900 & $-17.9 \pm 7.6$ \\
2 & 0.30173 & 900 & $-24.4 \pm 7.2$ \\
3 & 0.31455 & 900 & $-24.3 \pm 7.7$ \\
4 & 0.32915 & 900 & $-24.4 \pm 8.4$ \\
5 & 0.34372 & 1200 & $-22.3 \pm 7.7$ \\
6 & 0.35514 & 1200 & $-32.3 \pm 7.5$ \\
7 & 0.36276 & 1200 & $-33.3 \pm 7.6$ \\
8 & 0.37037 & 600 & $-28.8 \pm 6.4$ \\
9 & 0.37799 & 600 & $-30.2 \pm 7.3$ \\
10 & 0.38561 & 600 & $-13.9 \pm 7.9$ \\
11 & 0.39499 & 600 & $-25.7 \pm 7.8$ \\
12 & 0.40617 & 600 & $-41.5 \pm 7.6$ \\
& & & \\
\hline
\end{tabular}

Note. - Velocities are not absolute, but relative to a model atmosphere and include heliocentric corrections; see Section 3.1

TABLE 4

Observations and RAdial Velocity Data for LAWDS 7

\begin{tabular}{lccc}
\hline \hline $\begin{array}{l}\text { Exposure } \\
\text { Number }\end{array}$ & $\begin{array}{c}T_{\operatorname{mid}} \\
(\text { HJD-2454650.5) }\end{array}$ & $\begin{array}{c}t_{\exp } \\
(\mathrm{s})\end{array}$ & $\begin{array}{c}v_{\text {rel }} \\
\left(\mathrm{km} \mathrm{s}^{-1}\right)\end{array}$ \\
\hline 1 & 0.43018 & 900 & $-31.5 \pm 7.5$ \\
2 & 0.44304 & 1200 & $-18.8 \pm 7.0$ \\
3 & 0.45758 & 1200 & $-33.2 \pm 5.1$ \\
4 & 0.47215 & 1200 & $-39.9 \pm 7.6$ \\
5 & 0.48668 & 1200 & $-31.1 \pm 8.0$ \\
6 & 0.50141 & 1200 & $-24.3 \pm 5.4$ \\
7 & 0.51598 & 1200 & $-37.2 \pm 7.3$ \\
8 & 0.53051 & 1200 & $-26.4 \pm 3.6$
\end{tabular}

Note. - Velocities are not absolute, but relative to a model atmosphere and include heliocentric corrections; see Section 3.1

trum. We used the fxcor package in IRAF (Fitzpatrick 1993). We ran cross-correlations using a variety of continuum fitting techniques, Fourier filtering parameters, and wavelength ranges, including the central regions of the Balmer absorption lines, the entirety of the lines, and the entire spectrum. The final constraints described in the next paragraph resulted in radial velocity measurements that were the most self-consistent and stable against small changes in the task parameters.

We fit the observed and template spectra continua (see below) with a fourth-order cubic spline over wavelengths of $4180 \AA$ to $7500 \AA$. We rejected discrepant wavelength points using sigma- clipping rejection of $5 \sigma$ for points above the fit spline and $2 \sigma$ for points below the spline; we ran eight rejection iterations. This resulted in efficient exclusion of the broad Balmer absorption lines as well as noise from poor night sky subtraction and regions of spectral order overlap. Visual inspection of the normalized template spectra suggested an excellent continuum fit, while the normalized object spectra often had remaining low-frequency wiggles in the continuum.

${ }^{5}$ IRAF is distributed by the National Optical Astronomy Observatory, which is operated by the Association of Universities for Research in Astronomy (AURA) under a cooperative agreement with the National Science Foundation.
These fluctuations were highly resistant to efforts to remove them, and we were loathe to increase the order of the continuum fitting spline lest we inadvertently introduced significant artificial residuals into the Balmer line profiles.

To minimize the effects of this low-frequency residual in the continuum and to reduce any impact of cosmic ray hits or poor sky subtraction on the cross-correlation, we Fourier filtered the normalized spectrum using a ramp filter rising linearly from zero to one between Fourier wavenumbers $k_{1}$ and $k_{2}$, and falling linearly back to zero between wavenumbers $k_{3}$ and $k_{4}$. We selected $k_{1}=6$ and $k_{2}=9$ based on best practice suggestions by Wegner et al. (1999) and Alpaslan (2009). The low-frequency object spectrum continuum fitting residuals were effectively removed by this filtering. We set $k_{3}=3000$ (roughly one spectral resolution element) and $k_{4}=6000$ (roughly three pixels) to remove any highfrequency signals that could not be present in the intrinsic WD spectrum.

Finally, our cross-correlation focused on the spectral regions within $25 \AA$ of the rest-wavelength $\mathrm{H} \alpha$ and $\mathrm{H} \beta$ line centroids. Significantly larger spectral regions, such as including the entire absorption line, resulted in large and unstable changes in cross-correlation velocities with minor changes in fitting parameters, while smaller regions could exclude a signal from a high velocity amplitude binary.

We cross-correlated each spectrum with three different template spectra: the coadded ESI spectrum for each WD, the highest single signal-to-noise object spectrum of each WD, and the Koester model DA spectrum corresponding to the atmospheric parameters from Williams \& Bolte (2007). We resampled these model spectra to vacuum wavelengths and smoothed by a Gaussian kernel with FWHM equal to our spectral resolution. This final template option resulted in the smallest uncertainties in velocity as calculated by fxcor; these errors of $\approx 7 \mathrm{~km} \mathrm{~s}^{-1}$, were verified by the standard deviation of the measurements: $7.0 \mathrm{~km} \mathrm{~s}^{-1}$ and $6.5 \mathrm{~km} \mathrm{~s}^{-1}$ for LAWDS 4 and LAWDS 7, respectively, under the assumption of constant radial velocities. This uncertainty is also similar to our expectations based on the instrumental resolution and object spectrum signal-to-noise. We therefore chose to use the model spectra as our crosscorrelation templates.

The Koester model spectra predate the recent changes to physical parameters in model WD atmospheres, so we tested for potential effects of template mismatch by cross-correlating the observed spectra with models of significantly different $T_{\text {eff }}$ and $\log g$. We found systematic offsets of a few $\mathrm{km} / \mathrm{s}$, comparable in size to our stated measurement errors. Further, since the updated physics does not affect the rest wavelength of the line centers, and since these centers dominate the cross-correlation signal, we are confident that our conclusions would not change significantly with newer models.

The cross-correlation velocities are given in Table 3 for LAWDS 4 and Table 4 for LAWDS 7 ; these velocities are plotted in Figure 3. These are relative, not absolute, radial velocities. Relative heliocentric corrections reflecting the change in velocity over the observations have been applied, but gravitational redshifts and pressure 


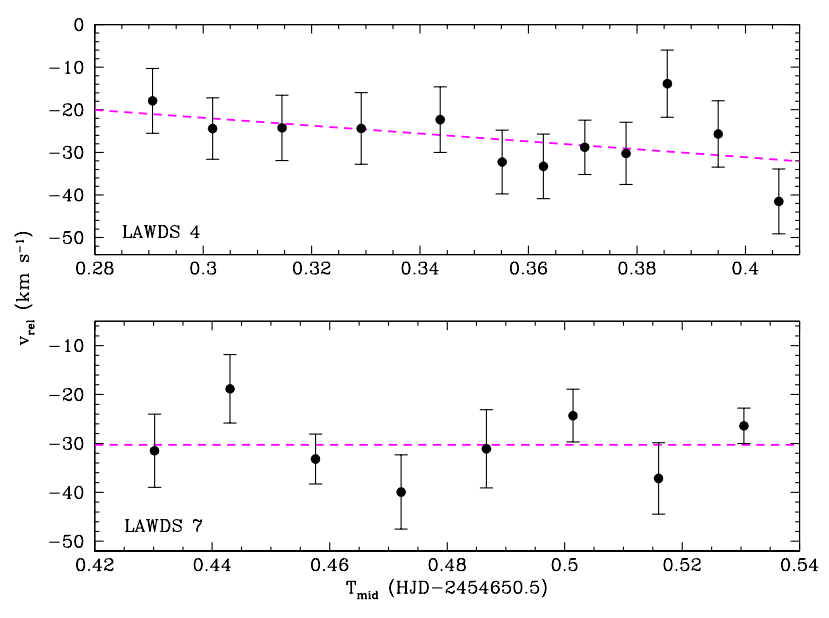

FIG. 3.- Relative velocities for LAWDS 4 (top) and LAWDS 7 (bottom) after cross-correlation with the best-fitting model spectra. The dashed magenta line indicates the best-fitting linear trend for LAWDS 4 and the mean velocity for LAWDS 7. Neither object shows evidence of high-amplitude, short-period radial velocity variations.

shifts of the $\mathrm{H} \beta$ line centroid (e.g., Grabowski et al. 1987; Halenka et al. 2015) have been ignored at this point, since any orbital motion will be reflected as a change in radial velocity, and the gravitational redshift and pressure shifts should not change sign or magnitude due to orbital motion.

Neither LAWDS 4 nor LAWDs 7 exhibits any evidence of high-amplitude short-period radial velocity variations. The individual velocity measurements for LAWDS 7 are completely consistent with the mean relative velocity of $v_{\text {rel }}=-30.3 \mathrm{~km} \mathrm{~s}^{-1}$ once the measurement errors are considered. LAWDS 4 may show some evidence of a negative velocity trend over the observations, with a formal slope of $-93 \pm 54 \mathrm{~km} \mathrm{~s}^{-1} \mathrm{~d}^{-1}$, though this slope is not statistically significant. If we assume that the observed slope is real and due to binary motion, the lack of any flattening or inflection of the radial velocity curve constrains any orbital period to be $>5.5 \mathrm{~h}$, twice the length of the observational series.

We determined the absolute radial velocities by measuring the centroid of the $\mathrm{H} \alpha$ NLTE line core in the coadded spectrum of each object, as this measurement is unaffected by pressure shifts (Koester 1987; Falcon et al. 2010; Halenka et al. 2015). Based on the spectral signal to noise $(\sim 100$ per resolution element for the summed spectra), we estimated the errors of the centroiding procedure to be $\approx 5.6 \mathrm{~km} \mathrm{~s}^{-1}$. The velocities were then corrected to heliocentric velocities and listed in Table 5 .

\section{DISCUSSION}

For each WD, we estimated the minimum expected radial velocity changes over the course of the observations by a DD Type Ia supernova progenitor system. If we assume that the total system mass is $1 M_{\mathrm{C} h}$ and the merger timescale is $14 \mathrm{Gyr}$, and if we assume that the observations are centered precisely on the maximum or minimum of the radial velocity curve, then we would expect a change in radial velocity of $\gtrsim 40 \sin i \mathrm{~km} \mathrm{~s}^{-1}$. Given the lack of significant velocity changes, LAWDS 4 and/or LAWDS 7 therefore can only be Type Ia super-
TABLE 5

CO-ADDED SPECTRUM VELOCITIES AND GRAVITATIONAL REDSHIFT CORRECTIONS

\begin{tabular}{cccc}
\hline \hline Object & $\begin{array}{c}v_{\mathrm{obs}} \\
\left(\mathrm{km} \mathrm{s}^{-1}\right)\end{array}$ & $\begin{array}{c}v_{\mathrm{GR}} \\
\left(\mathrm{km} \mathrm{s}^{-1}\right)\end{array}$ & $\begin{array}{c}v_{r} \\
\left(\mathrm{~km} \mathrm{~s}^{-1}\right)\end{array}$ \\
\hline LAWDS 4 & $36.1 \pm 5.6$ & $48.3 \pm 3.7$ & $-12.2 \pm 6.7$ \\
LAWDS 7 & $22.4 \pm 5.6$ & $58.3 \pm 4.4$ & $-35.9 \pm 7.1$
\end{tabular}

Note. - Velocities are solely for the H $\alpha$ NLTE line core to avoid uncertainties due to pressure shifts in the $\mathrm{H} \beta$ line. Errors in the gravitational redshift are calculated from mass uncertainties in Table 2 The errors in $v_{r}$ are these $v_{\text {obs }}$ and $v_{\mathrm{GR}}$ errors added in quadrature.

nova progenitor systems if we are observing a long-delay progenitor at low inclination and at the least favorable orbital phase.

Could LAWDS 4 and LAWDS 7 simply be wide binary systems in NGC 6633 with orbital timescales and velocity amplitudes not detectable in our one night of observations? Jeffries (1997) and Kharchenko et al. (2013) found the heliocentric radial velocity of NGC 6633 is $\approx-28 \mathrm{~km} \mathrm{~s}^{-1}$. In order to compare our radial velocities for LAWDS 4 and LAWDS 7 to the cluster velocity, we must correct the measured velocities for gravitational redshift.

As pointed out by, e.g., Falcon et al. (2010), the measured velocity $v_{\text {obs }}$ is the sum of a star's radial velocity $v_{r}$ and the gravitational redshift $v_{\mathrm{GR}}$; i.e., $v_{\mathrm{obs}}=v_{r}+v_{\mathrm{GR}}$. We calculate the predicted gravitational redshift from the revised $\log g$ and mass of each WD (see Section 2). The calculated $v_{\mathrm{GR}}$ and derived radial velocity $v_{r}$ for both WDs are given in Table 5.

The corrected WD heliocentric radial velocity of LAWDS 7 is consistent with the cluster radial velocity at the $1.1 \sigma$ level, while that of LAWDS 4 differs at the $2.5 \sigma$ level. Therefore, LAWDS 4 is unlikely to be a cluster member.

As for LAWDS 7, in Williams \& Bolte (2007) we noted that its color $(B-V=0.23 \pm 0.03)$ was too red based on the reddened best-fit spectral models $(B-V=0.15$ for our revised parameters in this paper). This mismatch, if significant, could be due to the presence of a cooler companion 6. Single WD model spectra can give satisfactory fits to composite WD spectra (Bergeron et al. 1989; Lajoie \& Bergeron 2007), and both $\mathrm{H} \alpha$ NLTE cores in a long-period or low-inclination binary should be near the same systemic velocity and thus indistinguishable at our spectral resolution, so we conservatively conclude that LAWDS 7 remains a viable candidate cluster member DD system.

Ultimately, parallax data from GAIA should be of sufficient quality to settle the question of binarity for both WDs. If one or both WDs are truly cluster members, over-luminous, and therefore likely binaries, this would support our initial conjecture that intermediate-age open clusters may indeed be fruitful hunting grounds for massive WD binaries.

6 The text of Williams \& Bolte (2007) incorrectly compared the model prediction to the photometry of LAWDS NGC 66336 . 
K.A.W. is grateful for the financial support of National Science Foundation award AST-0602288. This work was done in part through the REU Program in Physics and Astronomy at Texas A\&M University-Commerce funded by the National Science Foundation under grant PHY1359409. S.C. was supported in part by a Summer Research Assistant award from Texas A\&M UniversityCommerce. We thank S. Casewell, D. Koester, and S. Geier for useful insight provided in discussions at the 2014 and 2016 European White Dwarf Workshops. We also thank M. Wood for insightful discussions and his mastery of Python scripts used during analysis of some unfortunately spurious results. K.A.W. also wishes
P. Dobbie well, thanks him profusely for many important and insightful discussion, and will miss his careful and important contributions to the field. Keck telescope time was granted by NOAO, through the Telescope System Instrumentation Program (TSIP). TSIP is funded by NSF. The authors wish to recognize and acknowledge the very significant cultural role and reverence that the summit of Maunakea has always had within the indigenous Hawaiian community. We are most fortunate to have the opportunity to conduct observations from this mountain. This research has made use of NASA's Astrophysics Data System and of the SIMBAD database, operated at CDS, Strasbourg, France.

Facilities: Keck:II (ESI,TSIP)

\section{REFERENCES}

Alpaslan, M. 2009, arXiv:0912.4755

Barris, B. J., \& Tonry, J. L. 2006, ApJ, 637, 427

Bergeron, P., Saffer, R. A., \& Liebert, J. 1992, ApJ, 394, 228

Bergeron, P., Wesemael, F., Fontaine, G., \& Liebert, J. 1989, ApJ, 345, L91

Bergeron, P., Wesemael, F., Dufour, P., et al. 2011, ApJ, 737, 28

Catalán, S., Isern, J., García-Berro, E., \& Ribas, I. 2008, MNRAS, 387, 1693

Chakraborty, S. 2013, MS thesis, Texas A\&M University Commerce

Cummings, J. D., Kalirai, J. S., Tremblay, P.-E., \& Ramirez-Ruiz, E. 2015, ApJ, 807, 90

Dias, W. S., Monteiro, H., Caetano, T. C., et al. 2014, A\&A, 564, A79

Dobbie, P. D., Day-Jones, A., Williams, K. A., et al. 2012, MNRAS, 423, 2815

Dobbie, P. D., Napiwotzki, R., Burleigh, M. R., et al. 2009, MNRAS, 395, 2248

Duchêne, G., \& Kraus, A. 2013, ARA\&A, 51, 269

Falcon, R. E., Winget, D. E., Montgomery, M. H., \& Williams, K. A. 2010, ApJ, 712, 585

Finley, D. S., Koester, D., \& Basri, G. 1997, ApJ, 488, 375

Fitzpatrick, M. J. 1993, in Astronomical Society of the Pacific Conference Series, Vol. 52, Astronomical Data Analysis Software and Systems II, ed. R. J. Hanisch, R. J. V. Brissenden, \& J. Barnes, 472

Garcia-Berro, E., Soker, N., \& Althaus, L. G. 2015, NewA, in press (arXiv:1503.01739v2)

García-Berro, E., Torres, S., Renedo, I., et al. 2011, A\&A, 533 , A31

Geller, A. M., Hurley, J. R., \& Mathieu, R. D. 2013, AJ, 145, 8

Gianninas, A., Bergeron, P., \& Ruiz, M. T. 2011, ApJ, 743, 138

Grabowski, B., Halenka, J., \& Madej, J. 1987, ApJ, 313, 750

Greenstein, J. L., \& Peterson, D. M. 1973, A\&A, 25, 29

Halenka, J., Olchawa, W., Madej, J., \& Grabowski, B. 2015, ApJ, 808,131

Holberg, J. B., \& Bergeron, P. 2006, AJ, 132, 1221

Hurley, J. R., \& Shara, M. M. 2003, ApJ, 589, 179

Iben, Jr., I., \& Tutukov, A. V. 1984, ApJS, 54, 335

Jeffries, R. D. 1997, MNRAS, 292, 177

Jeffries, R. D., Totten, E. J., Harmer, S., \& Deliyannis, C. P. 2002, MNRAS, 336, 1109

Kalirai, J. S., Bergeron, P., Hansen, B. M. S., et al. 2007, ApJ, 671,748

Kalirai, J. S., Hansen, B. M. S., Kelson, D. D., et al. 2008, ApJ, 676,594

Kharchenko, N. V., Piskunov, A. E., Röser, S., Schilbach, E., \& Scholz, R.-D. 2005, A\&A, 438, 1163

Kharchenko, N. V., Piskunov, A. E., Schilbach, E., Röser, S., \& Scholz, R.-D. 2013, A\&A, 558, A53
Koester, D. 1987, ApJ, 322, 852

- 2010, Mem. Soc. Astron. Italiana, 81, 921

Koester, D., Kepler, S. O., Kleinman, S. J., \& Nitta, A. 2009,

Journal of Physics Conference Series, 172, 012006

Kowalski, P. M., \& Saumon, D. 2006, ApJ, 651, L137

Lajoie, C.-P., \& Bergeron, P. 2007, ApJ, 667, 1126

Liebert, J., Fontaine, G., Young, P. A., Williams, K. A., \& Arnett, D. 2013, ApJ, 769, 7

Liebert, J., Young, P. A., Arnett, D., Holberg, J. B., \& Williams, K. A. 2005, ApJ, 630, L69

Lucy, L. B., \& Ricco, E. 1979, AJ, 84, 401

Mannucci, F., Della Valle, M., Panagia, N., et al. 2005, A\&A, 433,807

Maoz, D., Mannucci, F., Li, W., et al. 2011, MNRAS, 412, 1508

Maoz, D., Mannucci, F., \& Nelemans, G. 2014, ARA\&A, 52, 107

Maoz, D., Sharon, K., \& Gal-Yam, A. 2010, ApJ, 722, 1879

Marigo, P., Girardi, L., Bressan, A., et al. 2008, A\&A, 482, 883

Monet, D. G., Levine, S. E., Canzian, B., et al. 2003, AJ, 125, 984

Munn, J. A., Monet, D. G., Levine, S. E., et al. 2004, AJ, 127, 3034

Napiwotzki, R., Christlieb, N., Drechsel, H., et al. 2001, Astronomische Nachrichten, 322, 411

Pinsonneault, M. H., \& Stanek, K. Z. 2006, ApJ, 639, L67

Press, W. H., Teukolsky, S. A., Vetterling, W. T., \& Flannery, B. P. 1992, Numerical recipes in FORTRAN. The art of scientific computing (New York: Cambridge University Press)

Prochaska, J. X., Gawiser, E., Wolfe, A. M., Cooke, J., \& Gelino, D. 2003, ApJS, 147, 227

Rubin, K. H. R., Williams, K. A., Bolte, M., \& Koester, D. 2008, AJ, 135, 2163

Santander-García, M., Rodríguez-Gil, P., Corradi, R. L. M., et al. 2015, Nature, 519, 63

Scannapieco, E., \& Bildsten, L. 2005, ApJ, 629, L85

Sheinis, A. I., Bolte, M., Epps, H. W., et al. 2002, PASP, 114, 851

Sullivan, M., Le Borgne, D., Pritchet, C. J., et al. 2006, ApJ, 648, 868

Tremblay, P.-E., \& Bergeron, P. 2009, ApJ, 696, 1755

Tremblay, P.-E., Bergeron, P., \& Gianninas, A. 2011, ApJ, 730, 128

Tremblay, P.-E., Ludwig, H.-G., Steffen, M., \& Freytag, B. 2013, A\&A, 552, A13

Wegner, G., Colless, M., Saglia, R. P., et al. 1999, MNRAS, 305, 259

Williams, K. A. 2002, PhD thesis, UNIVERSITY OF CALIFORNIA, SANTA CRUZ

Williams, K. A., \& Bolte, M. 2007, AJ, 133, 1490

Williams, K. A., Bolte, M., \& Koester, D. 2004, ApJ, 615, L49 -. 2009, ApJ, 693, 355

Zhao, J. K., Oswalt, T. D., Willson, L. A., Wang, Q., \& Zhao, G. 2012, ApJ, 746, 144 